\title{
OPTIMAL CONTROL FOR A CONTROLLED ILL-POSED WAVE EQUATION WITHOUT REQUIRING THE SLATER HYPOTHESIS ${ }^{1}$
}

\author{
Abdelhak Hafdallah \\ Laboratory of Mathematics, Informatics and Systems, University of Larbi Tébessi, \\ Rue de Constantine 12002, Tébessa, Algeria \\ abdelhak.hafdallah@univ-tebessa.dz
}

\begin{abstract}
In this paper, we investigate the problem of optimal control for an ill-posed wave equation without using the extra hypothesis of Slater i.e. the set of admissible controls has a non-empty interior. Firstly, by a controllability approach, we make the ill-posed wave equation a well-posed equation with some incomplete data initial condition. The missing data requires us to use the no-regret control notion introduced by Lions to control distributed systems with incomplete data. After approximating the no-regret control by a low-regret control sequence, we characterize the optimal control by a singular optimality system.
\end{abstract}

Keywords: Ill-posed wave equation, No-regret control, Incomplete data, Carleman estimates, Nullcontrollability.

\section{Introduction}

The first systematic study of optimal control of ill-posed problems was by J.L. Lions in his book "Control of distributed singular systems" [11], exactly when he focused on an ill-posed heat equation (backward heat equation). In his study, he required the set of admissible controls $U_{a d}$ to have a non-empty interior. This condition is the so-called Slater hypothesis. Regrettably, a difficulty starts when we need to use some sets like the positive cone $\left(L^{2}\right)^{+}$, which has an empty interior, as a set of admissible controls, where the hypothesis of Slater doesn't hold. To avoid such kind of obstacle, we propose to take a different approach to the regularization approach proposed in [3] and [5], to get an optimality system characterizing the optimal control without requiring Slater extra-hypothesis, it's the controllability approach.

The aim of our work is to generalize existing results $[3,5]$ where we seek to get an optimality system characterizing the optimal control for an ill-posed wave equation $[4,5]$, to reach our goal, we start by assuming that when taking the control in some dense space of $L^{2}(Q)$, the problem becomes well-posed. Then, by null-controllability of the well-posed wave equation, we seek to retrieve the second order time condition in the ill-posed equation. Hence, we get an optimal control problem for a controlled wave equation with incomplete data where we apply the no-regret control method introduced by Lions [12] (the original idea was introduced by Savage in [15]) for optimal control problems with incomplete data.

On the contrary of [5], this work leads us to characterize the optimal control by an optimality system which has a simpler form than the one given in [5], this will be very beneficial in a numerical analysis viewpoint.

\footnotetext{
${ }^{1}$ This work was supported by the Directorate-General for Scientific Research and Technological Development (DGRSDT).
} 
A few studies have been published in the context of optimal control of PDEs with missing data after that by Lions himself [13].

Later, many papers are published such as [14] and [9] where authors studied an age-structured population dynamics of incomplete data. In [1], authors applied the notion of no-regret control on a fractional wave equation with incomplete data. Afterward, a control coupled systems and a wave equation both with incomplete data were treated in [6] and [7] respectively, extended recently to more general and abstract systems in [8] .

Actually, the method of no-regret control consists of taking only controls $v$ such that

$$
J(v, g) \leq J(0, g),
$$

( $J$ is the cost function) for every missing data $g$, where we guarantee the belonging of the optimal control to this set of controls. To avoid the difficulty of characterizing the no-regret control, we should relax the definition by making a quadratic perturbation in no-regret control definition, i.e.

$$
J(v, g) \leq J(0, g)+\gamma\|g\|^{2}, \quad \gamma>0 .
$$

In this way, we define a sequence of low-regret controls expected to be converging to the noregret control.

The recent paper is organized as follows: in the next section we present some preliminaries for the main problem, in the third section we prove existence and uniqueness for the controllability problem, in the fourth we introduce the optimal control problem with missing data, in the fifth, we give an optimality system for the optimal control problem, and we finish with a conclusion.

\section{Preliminaries}

Let $\Omega \subset \mathbb{R}^{N}$ be an open bounded domain with smooth boundary $\Gamma, \Gamma_{0}$ is a non-empty subset of $\Gamma$, denote $Q=\Omega \times(0, T), \Sigma=\Gamma \times(0, T), \Sigma_{0}=\Gamma_{0} \times(0, T)$ and $T>0$. Consider the following wave equation given by:

$$
\begin{cases}y^{\prime \prime}-\triangle y=v & \text { in } Q \\ y(x, 0)=0, \quad y(x, T)=0 & \text { in } \Omega \\ y(x, t)=0 & \text { on } \Sigma\end{cases}
$$

where $v$ is a distributed control in

$$
U_{a d}^{\rho}=\left\{v \in L_{\rho}^{2}(Q): v \geq 0 \text { almost everywhere in } Q\right\},
$$

it's the closed convex cone $\left(L_{\rho}^{2}(Q)\right)^{+}$, where

$$
L_{\rho}^{2}(Q)=\left\{w \in L^{2}(Q) \text { such that } \rho w \in L^{2}(Q)\right\}
$$

and $\rho$ is a positive function defined on $Q$ such that $1 / \rho$ is bounded in $Q$. It's well known that (2.1) is ill-posed [5].

On the other hand, let's consider a null controllability problem for the following wave equation:

$$
\begin{cases}y^{\prime \prime}-\triangle y=v & \text { in } Q, \\ y(x, 0)=0, \quad y^{\prime}(x, 0)=g & \text { in } \Omega, \\ y(x, t)= \begin{cases}\theta & \text { on } \Sigma_{0}, \\ 0 & \text { on } \Sigma \backslash \Sigma_{0},\end{cases} \end{cases}
$$

where $g \in L^{2}(\Omega)$ is a missing initial condition. 
The following geometric and time conditions hold:

$$
\begin{gathered}
\exists x_{0} \notin \bar{\Omega} \text { such that }\left\{x \in \partial \Omega:\left(x-x_{0}\right) . \nu(x) \geq 0\right\} \subset \Gamma_{0}, \\
T>2 \sup _{x \in \Omega}\left|x-x_{0}\right|,
\end{gathered}
$$

and $\nu(x)$ denotes the external unit normal vector at $x$.

Note that for every $(v, g ; \theta) \in U_{a d}^{\rho} \times L^{2}(\Omega) \times L^{2}\left(\Sigma_{0}\right)$, the system (2.2)-(2.4) has a unique solution $y(v, g ; \theta)=y(v, g ; \theta)(x, t)$ in some sense (see [10, Ch. 4, p. 325]).

Actually, we want to find a function $\theta \in L^{2}\left(\Gamma_{0} \times(0, T)\right)$ such that for every $v \in U_{a d}^{\rho}$ and every missing initial condition $g \in L^{2}(\Omega)$ the solution of (2.2) verifies the following null controllability property

$$
y(x, T)=y^{\prime}(x, T)=0 .
$$

In this way, by the controllability of $(2.2)$ we retrieve the initial condition $y^{\prime}(x, 0)$ in $(2.1)$ but with an unknown value and (2.1) becomes a well-posed equation with missing data.

After this, we want to find a control function $v$ in $U_{a d}^{\rho}$ solution to the following optimal control problem

$$
\inf _{v \in U_{a d}^{\rho}} J_{\rho}(v, g) \quad \text { such that } \quad J_{\rho}(v, g)=\left\|y(v, g)-y_{d}\right\|_{\rho}^{2}+N\|v\|_{\rho}^{2},
$$

where $y_{d}$ is a target function in $L^{2}(Q), N>0$ all are given.

Now, let's prove the existence of a solution for the null-controllability problem (2.2)-(2.5).

\section{Existence for the null-controllability problem (2.2)-(2.5)}

Before treating the controllability problem (2.2)-(2.5) we announce the following theorem giving a so-called Carleman inequality type, which will be the main tool to solve the controllability problem.

Theorem 1. Denote the operator $L=\partial^{2} / \partial t^{2}-\Delta$ in distribution sense, under the geometric and time conditions (2.3)-(2.4) there exists a $C^{2}$ weighted positive function $\rho$ defined on $Q$ such that $1 / \rho$ is bounded in $Q$ and $C=C\left(\Omega, T, \Gamma_{0}, \rho\right)>0$ such that:

$$
\int_{0}^{T} \int_{\Omega} \frac{1}{\rho^{2}}|q|^{2} d x d t \leq C\left[\int_{0}^{T} \int_{\Omega} \frac{1}{\rho^{2}}|L q|^{2} d x d t+\int_{0}^{T} \int_{\Gamma_{0}} \frac{1}{\rho^{2}}\left|\frac{\partial q}{\partial \nu}\right|^{2} d \Gamma d t\right]
$$

for every

$$
q \in \mathcal{V}=\left\{\varphi \in L^{2}\left(0, T ; H_{0}^{1}(\Omega)\right): L \varphi \in L^{2}(Q),\left.\frac{\partial \varphi}{\partial \nu}\right|_{\Sigma_{0}} \in L^{2}\left(\Sigma_{0}\right)\right\}
$$

where

$$
L^{2}\left(0, T ; H_{0}^{1}(\Omega)\right)=\left\{\varphi:[0, T] \rightarrow H_{0}^{1}(\Omega) \text { measurable such that } \int_{0}^{T}\|\varphi(t)\|_{H_{0}^{1}(\Omega)}^{2} d t<\infty\right\} .
$$

P r o o f. It leads from a Carleman inequality, it can be found in [2, Theorem 1.1].

The inequality (3.1) allows us to introduce the following real inner product:

$$
a(r, s)=\int_{0}^{T} \int_{\Omega} \frac{1}{\rho^{2}} L r \cdot L s d x d t+\int_{0}^{T} \int_{\Gamma_{0}} \frac{1}{\rho^{2}} \frac{\partial r}{\partial \nu} \frac{\partial s}{\partial \nu} d \Gamma d t
$$

on $V$ the Hilbert space completion of $\mathcal{V}$, with its associated norm $\|\cdot\|_{a}=\sqrt{a(\cdot, \cdot)}$. 
Remark 1. We can characterize the structure of $\mathcal{V}$ as a subspace of a weighted Sobolev space. Indeed, let $H_{\rho}(Q)$ be the weighted Hilbert space defined by

$$
H_{\rho}(Q)=\left\{v \in L^{2}(Q) \text { such that: } \int_{0}^{T} \int_{\Omega} \frac{1}{\rho^{2}}|v|^{2} d x d t<\infty\right\},
$$

endowed with the natural norm

$$
\|\cdot\|_{H_{\rho}(Q)}=\left(\int_{0}^{T} \int_{\Omega} \frac{1}{\rho^{2}}|\cdot|^{2} d x d t\right)^{1 / 2} .
$$

This shows that $V$ is embedded continuously in $H_{\rho}(Q)$ as:

$$
\exists C>0:\|v\|_{H_{\rho}(Q)} \leq C\|v\|_{a} \quad \text { for every } \quad v \in V .
$$

By the boundedness of $1 / \rho^{2}$ on $Q$, we also see that $L^{2}(Q)$ is continuously embedded in $H_{\rho}(Q)$.

Proposition 1. Fix $(v, g) \in \mathrm{U}_{a d}^{\rho} \times L^{2}(\Omega)$. Define on $V$ the linear form

$$
l_{(v, g)}(s)=\int_{0}^{T} \int_{\Omega} v s d x d t+\int_{\Omega} g s(0) d x,
$$

then there exists a unique solution $\widetilde{p}(v, g) \in V$ to the following variational equation:

$$
a(r, s)=l_{(v, g)}(s), \quad \forall s \in V .
$$

Also, we have

$$
\exists C>0:\|\widetilde{p}(v, g)\|_{a} \leq C\left(\|v \rho\|_{L^{2}(Q)}+\|g\|_{L^{2}(\Omega)}\right) .
$$

Moreover, if we choose

$$
y(v, g)=\frac{1}{\rho^{2}} L \widetilde{p}(v, g), \quad \theta(v, g)=\left.\frac{1}{\rho^{2}} \frac{\partial \widetilde{p}(v, g)}{\partial \nu}\right|_{\Sigma_{0}},
$$

the pair $\{y(v, g), \theta(v, g)\}$ is a solution of the null controllability problem (2.2)-(2.5).

P r o o f. The result is obtained by application of the Lax-Milgram theorem with using Carleman inequality (3.1) to prove that the inner product (3.2) is coercive. Using (3.3) and integration by parts we get the null controllability property $(2.5)$.

\section{Optimal control of the controlled wave equation with incomplete data}

In this principal section, we focus on the following controlled wave equation missing initial condition

$$
\begin{cases}y^{\prime \prime}-\triangle y=v & \text { in } Q, \\ y(x, 0)=0, \quad y^{\prime}(x, 0)=g & \text { in } \Omega, \\ y= \begin{cases}\theta(v, g) & \text { on } \Sigma_{0}, \\ 0 & \text { on } \Sigma \backslash \Sigma_{0}\end{cases} \end{cases}
$$

with

$$
y(x, T)=0, \quad y^{\prime}(x, T)=0,
$$

where $\theta(v, g)$ is given by $(3.5)$. 
We solve the optimal control problem (4.1), (4.2), (2.6) regardless of the values of the missing initial condition $g$, where $L_{\rho}^{2}(Q)$ endowed with the inner product $(\cdot, \cdot)_{\rho}=(\rho \cdot, \rho \cdot)_{L^{2}(Q)}$ and the associated norm $\|\cdot\|_{\rho}=\sqrt{(\cdot, \cdot)_{\rho}}$.

In order to ensure the existence of the optimal control for (4.1), (4.2), (2.6) we need an extra hypothesis of Slater (see [11, Ch. 4, Remark 1.4]) which requires that

$$
U_{a d}^{\rho} \quad \text { has a non-empty interior. }
$$

Unfortunately, the extra hypothesis (4.3) is not fulfilled by $U_{a d}^{\rho}$ because it's well known that $\left(L^{2}(Q)\right)^{+}$has an empty interior.

However, we propose an approach where there is no need to (4.3), it's the method of no-regret control which was introduced by J.L. Lions in [12], to solve optimal control problems with some incomplete data.

First of all, let's give a definition of the no-regret control for the controlled system with missing data (4.1), (4.2), (2.6).

Definition 1 [12]. We say that $u \in U_{a d}^{\rho}$ is a no-regret control for (4.1), (4.2), (2.6) if $u$ is the solution of:

$$
\inf _{v \in U_{a d}^{\rho}}\left(\sup _{g \in L^{2}(\Omega)}\left(J_{\rho}(v, g)-J_{\rho}(0, g)\right)\right)
$$

In the following lemma, we try to rewrite the main quantity in the last definition to isolate the missing data in some way.

Lemma 1. Let $M$ be an operator defined from $L^{2}(\Omega)$ to $L^{2}\left(\Sigma_{0}\right)$ by $M g=\frac{\partial \widetilde{p}}{\partial \nu}(0, g)$, where $\widetilde{p}(v, g)$ is the unique solution to (3.3). Then, $M$ is a linear bounded operator on $L^{2}(\Omega)$, and we have

$$
J_{\rho}(v, g)-J_{\rho}(0, g)=J_{\rho}(v, 0)-J_{\rho}(0,0)+2(S(v), g)_{L^{2}(\Omega)},
$$

where $S$ is also a linear bounded operator from $U_{a d}^{\rho}$ to $L^{2}(\Omega)$ given by

$$
S(v)=\widetilde{p}(v, 0)(0)-M^{*}(\theta(v, 0)) .
$$

P r o o f. It's clear that $M$ is linear, also $M$ is bounded. In fact, we know that $\widetilde{p}(v, 0)$ solves $(3.3)$ for every $s \in V$, we choose an $s$ such that

$$
\begin{cases}L s=0 & \text { in } Q, \\ s(x, 0)=g, \quad s^{\prime}(x, 0)=0 & \text { in } \Omega, \\ \frac{\partial s}{\partial \nu}= \begin{cases}\frac{\partial \widetilde{p}(0, g)}{\partial \nu} & \text { on } \Sigma_{0}, \\ 0 & \text { on } \Sigma \backslash \Sigma_{0}\end{cases} \end{cases}
$$

to get

$$
\inf _{\Sigma_{0}} \frac{1}{\rho^{2}} \int_{0}^{T} \int_{\Gamma_{0}}\left|\frac{\partial \widetilde{p}(0, g)}{\partial \nu}\right|^{2} d \Gamma d t \leq \int_{0}^{T} \int_{\Gamma_{0}} \frac{1}{\rho^{2}}\left|\frac{\partial \widetilde{p}(0, g)}{\partial \nu}\right|^{2} d \Gamma d t=\|g\|_{L^{2}(\Omega)}^{2}
$$

From linearity in $(3.3)$, we get $y(v, g)=y(v, 0)+y(0, g)+y(0,0)$, and by a simple calculation we get

$$
J_{\rho}(v, g)-J_{\rho}(0, g)=J_{\rho}(v, 0)-J_{\rho}(0,0)+2(y(v, 0), y(0, g))_{\rho} .
$$


Use Green formula to prove

$$
\begin{gathered}
(y(v, 0), y(0, g))_{\rho}=(L \widetilde{p}(v, 0), y(0, g))_{L^{2}(Q)} \\
=(\widetilde{p}(v, 0), L y(0, g))_{L^{2}(Q)}-\left(\frac{\partial \widetilde{p}(v, 0)}{\partial \nu}, \theta(0, g)\right)_{L^{2}\left(\Sigma_{0}\right)}+(g, \widetilde{p}(v, 0)(0))_{L^{2}(\Omega)} \\
=(\widetilde{p}(v, 0)(0), g)_{L^{2}(\Omega)}-\left(\frac{\partial \widetilde{p}(v, 0)}{\partial \nu}, \frac{1}{\rho^{2}} \frac{\partial \widetilde{p}}{\partial \nu}(0, g)\right)_{L^{2}\left(\Sigma_{0}\right)} \\
=(\widetilde{p}(v, 0)(0), g)_{L^{2}(\Omega)}-\left(M^{*}(\theta(v, 0)), g\right)_{L^{2}(\Omega)} .
\end{gathered}
$$

We know that $\theta(v, 0): U_{a d}^{\rho} \rightarrow L^{2}\left(\Sigma_{0}\right)$ solves (3.4), choose $s=\widetilde{p}(v, 0)$ and use (4.2) to find

$$
\begin{gathered}
\inf _{\Sigma_{0}} \frac{1}{\rho^{2}} \int_{0}^{T} \int_{\Gamma_{0}}\left|\frac{\partial \widetilde{p}(v, 0)}{\partial \nu}\right|^{2} d \Gamma d t \leq \int_{0}^{T} \int_{\Gamma_{0}} \frac{1}{\rho^{2}}\left|\frac{\partial \widetilde{p}(v, 0)}{\partial \nu}\right|^{2} d \Gamma d t \leq \int_{0}^{T} \int_{\Omega} v \widetilde{p}(v, 0) d x d t \\
\leq\|v\|_{\rho}\|\widetilde{p}(v, 0)\|_{H_{\rho}(Q)} \leq C\|v\|_{\rho}^{2},
\end{gathered}
$$

which proves that $\theta(v, 0)$ is bounded. Moreover, the map $\widetilde{p}(v, 0)(0): U_{a d}^{\rho} \rightarrow L^{2}(\Omega)$ is continuous. In fact, by a Carleman estimate given in [2, Corollary 2.8], under the same condition of Theorem 1 there exists a $C^{2}$ weighted positive function $\rho$ on $Q$ such that $1 / \rho$ is bounded in $Q$ and $C=C\left(\Omega, T, \Gamma_{0}, \rho\right)>0$ such that:

$$
\int_{\Omega} \frac{1}{\rho(0)^{2}}|q(0)|^{2} d x d t \leq C\left[\int_{0}^{T} \int_{\Omega} \frac{1}{\rho^{2}}|L q|^{2} d x d t+\int_{0}^{T} \int_{\Gamma_{0}} \frac{1}{\rho^{2}}\left|\frac{\partial q}{\partial \nu}\right|^{2} d \Gamma d t\right]
$$

for every $q \in \mathcal{V}$. Choose $q=\widetilde{p}(v, 0)$ to find

$$
\int_{\Omega} \frac{1}{\rho(0)^{2}}|\widetilde{p}(v, 0)(0)|^{2} d x d t \leq C\left[\int_{0}^{T} \int_{\Omega} \frac{1}{\rho^{2}}|L \widetilde{p}(v, 0)|^{2} d x d t+\|\theta(v, 0)\|_{L^{2}\left(\Sigma_{0}\right)}^{2}\right] \leq C\|v\|_{\rho}^{2} .
$$

Finally, $S$ is also a linear bounded operator.

Unfortunately, we encounter a big difficulty when characterizing the no-regret control where we need to know the structure of the set

$$
\left\{v \in U_{a d}^{\rho}:(S(v), g)_{L^{2}(\Omega)}=0 \text { for every } g \text { in } L^{2}(\Omega)\right\},
$$

which is difficult to do, this requires on us to relax no-regret control definition by making some quadratic perturbation, then, we announce:

Definition 2 [12]. We say that $u_{\gamma} \in U_{a d}^{\rho}$ is a low-regret control for (4.1), (4.2), (2.6) if $u_{\gamma}$ is the solution of the problem:

$$
\inf _{v \in U_{a d}^{\rho}}\left(\sup _{g} \in L^{2}(\Omega)\left(J_{\rho}(v, g)-J_{\rho}(0, g)-\gamma\|g\|_{L^{2}(\Omega)}^{2}\right)\right), \quad \gamma>0 .
$$

From (4.5), we get for all $v \in U_{a d}^{\rho}$

$$
\begin{gathered}
\sup _{g \in L^{2}(\Omega)}\left(J_{\rho}(v, g)-J_{\rho}(0, g)-\gamma\|g\|_{L^{2}(\Omega)}^{2}\right) \\
=J_{\rho}(v, 0)-J_{\rho}(0,0)+\sup _{g \in L^{2}(\Omega)}\left(2(S(v), g)_{L^{2}(\Omega)}-\gamma\|g\|_{L^{2}(\Omega)}^{2}\right) \\
=J_{\rho}(v, 0)-J_{\rho}(0,0)+\frac{1}{\gamma}\|S(v)\|_{L^{2}(\Omega)}^{2} .
\end{gathered}
$$


Thus, our optimal control problem is transformed into a standard optimal control problem (i.e. a problem with complete data) given by

$$
\inf _{v \in U_{a d}^{\rho}} \mathcal{J}_{\rho}^{\gamma}(v),
$$

where

$$
\mathcal{J}_{\rho}^{\gamma}(v)=J_{\rho}(v, 0)-J_{\rho}(0,0)+\frac{1}{\gamma}\|S(v)\|_{L^{2}(\Omega)}^{2}
$$

Lemma 2. The problem (4.1), (4.2), (2.6), (4.6), (4.7) has a unique solution $u_{\gamma} \in U_{a d}^{\rho}$.

P r o o f. We have for every $v \in \mathcal{U}_{a d}^{\rho}: \mathcal{J}_{\rho}^{\gamma}(v) \geq-J_{\rho}(0,0)=-\left\|y_{d}\right\|_{\rho}^{2}$ then $d_{\gamma}=\inf _{v \in \mathcal{U}_{a d}^{\rho}} \mathcal{J}_{\rho}^{\gamma}(v)$ exists. Let $\left(v_{n}^{\gamma}\right)$ be a minimizing sequence such that $d_{\gamma}=\lim _{n \rightarrow \infty} \mathcal{J}_{\rho}^{\gamma}\left(v_{n}^{\gamma}\right)$. We know that

$$
\mathcal{J}_{\rho}^{\gamma}\left(v_{n}^{\gamma}\right)=J_{\rho}\left(v_{n}^{\gamma}, 0\right)-J_{\rho}(0,0)+\frac{1}{\gamma}\left\|S\left(v_{n}^{\gamma}\right)\right\|_{L^{2}(\Omega)}^{2} \leq d_{\gamma}+1 .
$$

This implies the following bounds

$$
\left\|v_{n}^{\gamma}\right\|_{\rho} \leq C_{\gamma}, \quad\left\|y\left(v_{n}^{\gamma}, 0\right)\right\|_{\rho} \leq C_{\gamma}, \quad \frac{1}{\sqrt{\gamma}}\left\|S\left(v_{n}^{\gamma}\right)\right\|_{L^{2}(\Omega)} \leq C_{\gamma},
$$

where $C_{\gamma}$ is a positive constant independent of $n$. Then, there exists $u_{\gamma}$ such that $v_{n}^{\gamma} \rightarrow u_{\gamma}$ weakly in $U_{a d}^{\rho}$ (closed), also $y\left(v_{n}^{\gamma}, 0\right) \rightarrow y\left(u_{\gamma}, 0\right)$ weakly in $L_{\rho}^{2}(Q)$ because of continuity w.r.t. the data.

Since $S$ is bounded, then

$$
S\left(v_{n}^{\gamma}\right) \rightarrow S\left(u_{\gamma}\right) \text { weakly in } L^{2}(\Omega),
$$

with

$$
\mathcal{J}_{\rho}^{\gamma}\left(u_{\gamma}\right) \leq \liminf _{n \rightarrow \infty} \mathcal{J}_{\rho}^{\gamma}\left(v_{n}^{\gamma}\right)
$$

and we conclude that

$$
\mathcal{J}_{\rho}^{\gamma}\left(u_{\gamma}\right)=\inf _{v \in U_{a d}^{\rho}} \mathcal{J}_{\rho}^{\gamma}(v) .
$$

Since $\mathcal{J}_{\rho}^{\gamma}(v)$ is strictly convex, $u_{\gamma}$ is unique.

It still remains to obtain an optimality system giving a characterization for low-regret control $u_{\gamma}$ as follows

Theorem 2. The low-regret control $u_{\gamma} \in U_{a d}^{\rho}$ which is a solution to (4.1), (4.2), (2.6), (4.6), (4.7) is characterized by the following optimality system

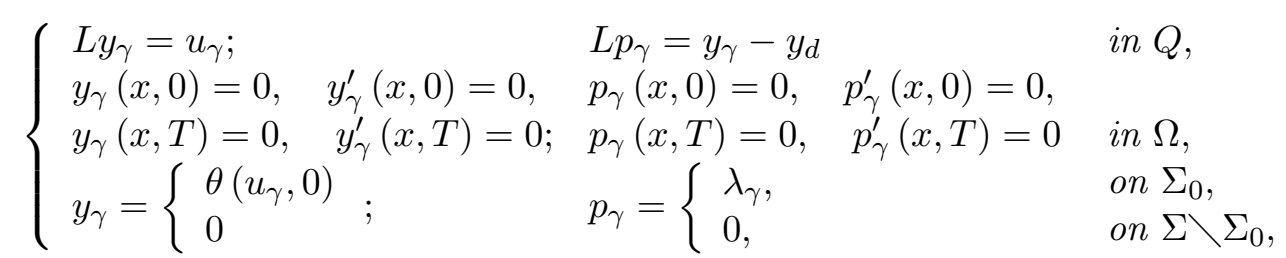

where $\gamma>0, y_{\gamma}=y\left(u_{\gamma}, 0\right)$ and $p_{\gamma}=p\left(u_{\gamma}\right)$, with the following variational inequality

$$
\left(T^{*}\left(L p_{\gamma}\right)+N u_{\gamma}+\frac{1}{\gamma} S^{*} S\left(u_{\gamma}\right), v-u_{\gamma}\right)_{\rho} \geq 0 \quad \forall v \in U_{a d}^{\rho},
$$

where $T: v \rightarrow y(v, 0)$ from $U_{a d}^{\rho}$ to $L_{\rho}^{2}(Q)$ is a linear bounded operator. 
P r o o f. A first order necessary condition of Euler-Lagrange [10] for (4.6), (4.7) gives for every $v \in U_{a d}^{\rho}$

$$
\left(y\left(u_{\gamma}, 0\right)-y_{d}, y\left(v-u_{\gamma}, 0\right)\right)_{\rho}+N\left(u_{\gamma}, v-u_{\gamma}\right)_{\rho}+\frac{1}{\gamma}\left(S\left(u_{\gamma}\right), S\left(v-u_{\gamma}\right)\right)_{L^{2}(\Omega)} \geq 0 .
$$

Denote $y_{\gamma}=y\left(u_{\gamma}, 0\right)$ and let $\sigma_{\gamma}=\sigma\left(u_{\gamma}\right)$ be the unique solution of the following variational equation

$$
a\left(\sigma_{\gamma}, q\right)=\int_{Q}\left(y_{\gamma}-y_{d}\right) q d x d t \quad \forall q \in V .
$$

Consider the pair $\left(p_{\gamma}, \lambda_{\gamma}\right)$ given by

$$
p_{\gamma}=\frac{1}{\rho^{2}} L \sigma_{\gamma}, \quad \lambda_{\gamma}=\left.\frac{1}{\rho^{2}} \frac{\partial \sigma_{\gamma}}{\partial \nu}\right|_{\Sigma_{0}},
$$

then $\left(p_{\gamma}, \lambda_{\gamma}\right)$ is the solution of the following backward wave equation

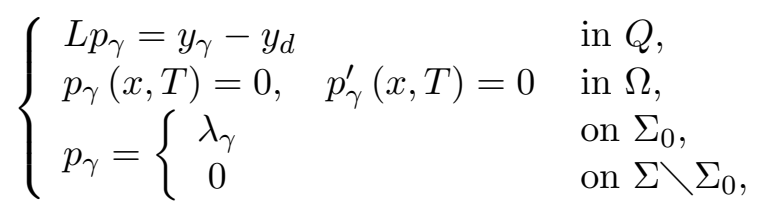

with the null controllability propriety

$$
p_{\gamma}(x, 0)=p_{\gamma}^{\prime}(x, 0)=0 .
$$

Rewrite the optimality condition (4.10) to be in the following form

$$
\left(L p_{\gamma}, y\left(v-u_{\gamma}, 0\right)\right)_{\rho}+\left(N u_{\gamma}+\frac{1}{\gamma} S^{*} S\left(u_{\gamma}\right), v-u_{\gamma}\right)_{\rho} \geq 0 \quad \forall v \in U_{a d}^{\rho}
$$

where

$$
\left(L p_{\gamma}, y\left(v-u_{\gamma}, 0\right)\right)_{\rho}=\left(T^{*}\left(L p_{\gamma}\right), v-u_{\gamma}\right)_{\rho} \quad \forall v \in U_{a d}^{\rho},
$$

which gives optimality condition (4.9).

The boundedness of $T$ follows from the continuity of the solution to (2.2), (2.5) w.r.t. data.

\section{No-regret control optimality system (Optimal control for the ill-posed wave equation)}

In this section, we will give an optimality system characterizing the optimal control (or the no-regret control) solution to (4.1), (4.2), (2.6), (4.4) by taking the limits of $u_{\gamma}, y_{\gamma}, p_{\gamma}, \theta\left(u_{\gamma}, 0\right)$ and $\lambda_{\gamma}$ when $\gamma \rightarrow 0$.

Theorem 3. There exists a positive constant $C$ independent of $\gamma$ such that

$$
\begin{gathered}
\left\|u_{\gamma}\right\|_{\rho} \leq C, \quad\left\|y_{\gamma}-y_{d}\right\|_{\rho} \leq C, \quad\left\|y_{\gamma}\right\|_{\rho} \leq C, \quad\left\|S\left(u_{\gamma}\right)\right\|_{L^{2}(\Omega)} \leq C \sqrt{\gamma}, \\
\left\|\sigma_{\gamma}\right\|_{\rho} \leq C, \quad\left\|\theta\left(u_{\gamma}, 0\right)\right\|_{L^{2}\left(\Sigma_{0}\right)} \leq C, \quad\left\|\lambda_{\gamma}\right\|_{L^{2}\left(\Sigma_{0}\right)} \leq C, \\
\left\|p_{\gamma}\right\|_{L^{\infty}\left(0, T ; H_{0}^{1}(\Omega)\right)} \leq C, \quad\left\|p_{\gamma}^{\prime}\right\|_{L^{\infty}\left(0, T ; L^{2}(\Omega)\right)} \leq C .
\end{gathered}
$$


P r o o f. Let $u_{\gamma}$ is the unique solution for (4.1), (4.2), (2.6), (4.6), (4.7), then

$$
\mathcal{J}_{\rho}^{\gamma}\left(u_{\gamma}\right) \leq \mathcal{J}_{\rho}^{\gamma}(0)=0 \text { i.e. } J_{\rho}\left(u_{\gamma}, 0\right)+\frac{1}{\gamma}\left\|S\left(u_{\gamma}\right)\right\|_{L^{2}(\Omega)}^{2} \leq J_{\rho}(0,0)
$$

which give (5.1a).

Choose $q=\sigma_{\gamma}$ in (4.11) with Lax-Milgram theorem stability estimates to prove that

$$
\exists C>0:\left\|\sigma_{\gamma}\right\|_{\rho} \leq C,
$$

where $C$ is independent of $\gamma$. From continuity and (5.1a), we deduce the boundedness of $\theta\left(u_{\gamma}, 0\right)$ and $\lambda_{\gamma}$ in $L^{2}\left(\Sigma_{0}\right)$.

Multiply (4.12) by $p_{\gamma}^{\prime}$, integrate by parts, and use (5.1a) with (5.1b) to find

$$
\left\|p_{\gamma}^{\prime}\right\|_{L^{\infty}\left(0, T ; L^{2}(\Omega)\right)}^{2}+\left\|p_{\gamma}\right\|_{L^{\infty}\left(0, T ; H_{0}^{1}(\Omega)\right)}^{2} \leq C\left(\left\|y_{\gamma}-y_{d}\right\|_{L^{2}(Q)}^{2}+\left\|\lambda_{\gamma}\right\|_{L^{2}\left(\Sigma_{0}\right)}^{2}\right) \leq C .
$$

Lemma 3. The low-regret control $u_{\gamma}$ solution to (4.1), (4.2), (2.6), (4.6), (4.7) converges in $U_{a d}^{\rho}$ to the no-regret control $u$ solution to (4.1), (4.2), (2.6), (4.4).

P r o o f. By (5.1a), we have

$$
\left\|L y_{\gamma}\right\|_{\rho} \leq C
$$

and

And by (5.1b) we have

$$
\begin{gathered}
u_{\gamma} \rightarrow u \quad \text { weakly in } U_{a d}^{\rho}, \\
y_{\gamma} \rightarrow y \quad \text { weakly in } L_{\rho}^{2}(Q) .
\end{gathered}
$$

$$
\theta\left(u_{\gamma}, 0\right) \rightarrow \theta(u, 0) \quad \text { weakly in } L^{2}\left(\Sigma_{0}\right) .
$$

We conclude that $y$ solves

$$
\begin{cases}L y=u & \text { in } Q \\ y(0)=0, \quad y^{\prime}(0)=0 & \text { in } \Omega \\ y(T)=0, \quad y^{\prime}(T)=0 & \text { in } \Omega \\ y= \begin{cases}\theta(u, 0) & \text { on } \Sigma_{0} \\ 0 & \text { on } \Sigma \backslash \Sigma_{0}\end{cases} \end{cases}
$$

Again, from (5.1a)

$$
S\left(u_{\gamma}\right) \rightarrow 0 \quad \text { strongly in } L^{2}(\Omega),
$$

then $(S(u), g)_{L^{2}(\Omega)}=0$ for every $g$ in $L^{2}(\Omega)$, which means that $u$ is a no-regret control solution to $(4.1),(4.2),(2.6),(4.4)$.

Finally, we can announce the following our main theorem characterizing the optimal control for the ill-posed wave equation (4.2).

Theorem 4. The no-regret control $u \in U_{a d}^{\rho}$ solution to (4.1), (4.2), (2.6), (4.5) is characterized by the following optimality system

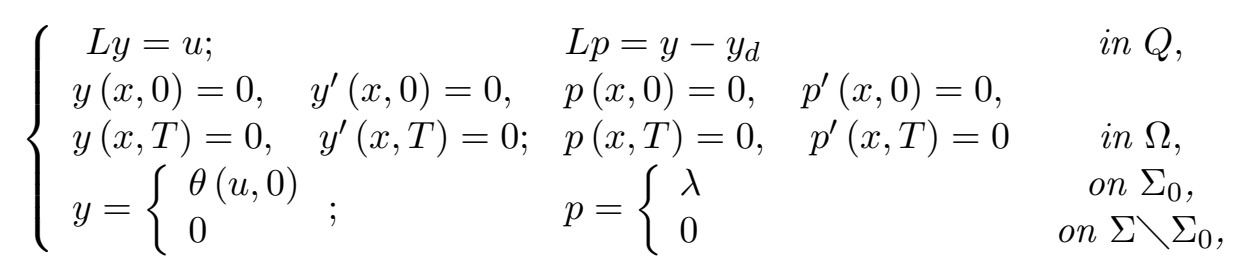




$$
\begin{aligned}
& \text { where } \lambda=\lim _{\gamma \rightarrow 0} \lambda_{\gamma}, y=y(u, 0) \text { and } p=p(u) \text {, with the following variational inequality } \\
& \qquad\left(T^{*}(L p)+N \rho^{2} u+S^{*} S(u), v-u\right)_{L^{2}(Q)} \geq 0 \quad \forall v \in U_{a d}^{\rho} .
\end{aligned}
$$

$\mathrm{P} \mathrm{r}$ o o f. We have already proved the convergence of $y_{\gamma}$ to $y$, and $u_{\gamma}$ to $u$ in the proof of Lemma 3. For the rest, use (5.1a) to get

$$
\left\|L p_{\gamma}\right\|_{\rho} \leq C
$$

and (5.1b), to find

$$
\lambda_{\gamma} \rightarrow \lambda \text { weakly in } L^{2}\left(\Sigma_{0}\right),
$$

Passing to the limit when $\gamma \rightarrow 0$ in (4.8) we obtain the optimality system (5.2).

\section{Conclusion}

To sum up, our work leads to solving the optimal control problem for an ill-posed wave equation without requiring the extra hypothesis of Slater. The main idea was to make a null controllability approach to deal with a well-posed equation with a missing initial condition. Then, we have applied the no-regret control method to solve the optimal control with incomplete data. The optimality system describing the optimal control is built by an overdetermined optimal state and adjoint state.

\section{Acknowledgements}

The author wish to acknowledge the editor and anonymous reviewers for their insightful comments, which have improved the quality of this publication.

\section{REFERENCES}

1. Baleanu D., Joseph C., Mophou G. Low-regret control for a fractional wave equation with incomplete data. Adv. Difference Equ., 2016. Vol. 2016. Art. no. 240. P. 1-20. DOI: 10.1186/s13662-016-0970-8

2. Baudouin L., De Buhan M. and Ervedoza S. Global Carleman estimates for waves and applications. Comm. Partial Differential Equations, 2013. Vol. 38, No. 5. P. 823-859. DOI: $10.1080 / 03605302.2013 .771659$

3. Berhail A., Omrane A. Optimal control of the ill-posed Cauchy elliptic problem. Int. J. Differ. Equ., 2015. Vol. 2018. Art. no. 468918. P. 1-9. DOI: 10.1155/2015/468918

4. Ciprian G., Gal. N. J. A spectral approach to ill-posed problems for wave equations. Ann. Mat. Pura Appl. (4), 2008. Vol. 187, No. 4. P. 705-717. DOI: 10.1007/s10231-007-0063-0

5. Hafdallah A., Ayadi A., Laouar C. No-regret optimal control characterization for an illposed wave equation. Int. J. Math. Trends Tech., 2017. Vol. 45, No. 3. P. 283-287. DOI: $10.14445 / 22315373 /$ IJMTT-V41P528

6. Hafdallah A., Ayadi A. Optimal control of electromagnetic wave displacement with an unknown velocity of propagation. Int. J. Control, 2019. Vol. 92, No. 11. P. 2693-2700. (Published online: 2018) DOI: $10.1080 / 00207179.2018 .1458157$

7. Hafdallah A., Ayadi A. Optimal control of a thermoelastic body with missing initial conditions. Int. J. Control, 2020. Vol. 93, No. 7. P. 1570-1576. (Published online: 2018) DOI: $10.1080 / 00207179.2018 .1519258$

8. Hafdallah A. On the optimal control of linear systems depending upon a parameter and with missing data. Nonlinear Stud., 2020. Vol. 27, No. 2. P. 457-469.

9. Jacob B., Omrane A. Optimal control for age-structured population dynamics of incomplete data. J. Math. Anal. Appl., 2010. Vol. 370, No. 1. P. 42-48. DOI: 10.1016/j.jmaa.2010.04.042 
10. Lions J. L. Optimal Control of Systems Governed by Partial Differential Equations. Grundlehren Math. Wiss., vol. 170. Berlin, Heidelberg: Springer-Verlag, 1971. 400 p.

11. Lions J. L. Control of Distributed Singular Systems. Paris: Gauthier-Villars, 1985. 552 p.

12. Lions J. L. Contrôle à moindres regrets des systèmes distribués. C. R. Acad. Sci. Paris. Sér. I, Math., 1992. Vol. 315, No. 12. P. 1253-1257.

13. Lions J.L. No-Regret and Low-Regret Control, in Environment, Economics and Their Mathematical Models. Paris: Masson, 1994.

14. Nakoulima O., Omrane A., Velin J. On the Pareto control and no-regret control for distributed systems with incomplete data. SIAM J. Control Optim., 2003. Vol. 42, No. 4. P. 1167-1184. DOI: $10.1137 /$ S0363012900380188

15. Savage L. J. The Foundations of Statistics. 2nd ed. New York: Dover Publ., 1972. 310 p. 